\title{
Differential efficacy between stenting and plain balloon angioplasty for femoropopliteal disease with or without total occlusion
}

\author{
In-Ho Chae ${ }^{1}$, Chang-Hwan Yoon ${ }^{1}$, Young-Guk Ko ${ }^{2}$, Pil-Ki Min³ ${ }^{3}$ Jae-Hwan Lee ${ }^{4}$, Cheol Woong Yu, \\ Seung Whan Lee ${ }^{6}$, Sang-Rok Lee ${ }^{7}$, Seung Hyuk Choi ${ }^{8}$, Yoon Seok $\mathrm{Koh}^{9}$, Donghoon $\mathrm{Choi}^{2}$, and on behalf \\ of the K-VIS Investigators
}

\begin{abstract}
Division of Cardiology, Department of Internal Medicine, ${ }^{1}$ Seoul National University Bundang Hospital, Seoungnam; ${ }^{2}$ Severance Cardiovascular Hospital, Yonsei University College of Medicine, Seoul; ${ }^{3}$ Gangnam Severance Hospital, Yonsei University College of Medicine, Seoul; ${ }^{4}$ Chungnam National University Hospital, Daejon; ${ }^{5}$ Korea University Anam Hospital, Seoul; ${ }^{6}$ Asan Medical Center, University of Ulsan College of Medicine, Seoul; ${ }^{7}$ Jeonbuk National University Hospital, Jeonju; ${ }^{8}$ Samsung Medical Center, Sungkyunkwan University School of Medicine, Seoul; ${ }^{\text {Seoul St. Mary's }}$ Hospital, College of Medicine, The Catholic University of Korea, Seoul, Korea
\end{abstract}

Received: January 25, 2019 Revised : March 26, 2019 Accepted: April 2, 2019

\section{Correspondence to} Chang-Hwan Yoon, M.D. Department of Internal Medicine, Cardiovascular Center, Seoul National University Bundang Hospital, 82 Gumi-ro 173beon-gil, Bundang-gu, Seongnam 13620, Korea Tel: $+82-31-787-7052$ Fax: $+82-31-787-4052$ E-mail: changhwanyoon@gmail.com https://orcid.org/0000-00016305-4442
Background/Aims: Whether the presence of chronic total occlusion (CTO) affects patency after stenting in femoropopliteal lesions is unknown. We determined the effects of plain balloon angioplasty (POBA) in comparison with those of stenting on patency for femoropopliteal CTO and stenosis (non-CTO).

Methods: We analyzed data from the Korean Vascular Intervention Society Endovascular Therapy in Lower-Limb Artery Diseases Registry, a multicenter cohort of patients with lower extremity peripheral arterial disease. Data from 1,329 patients and 1558 limbs treated with endovascular intervention for at least one femoropopliteal lesion were evaluated.

Results: Among the 1,558 limbs, 345, 432, 275, and 506 were in the non-CTO-PO$\mathrm{BA}$, non-CTO-stent, CTO-POBA, and CTO-stent groups, respectively. During follow-up, loss of clinical primary patency, a composite of freedom from restenosis or clinically driven target lesion revascularization, occurred in 65 (18.8\%), 68 (15.7\%), 62 (22.5\%), and 113 limbs (22.3\%) in the non-CTO-POBA, non-CTO-stent, CTO-POBA, and CTO-stent groups, respectively. The patients in the non-CTOstent group showed a significantly better clinical primary patency than those in the no-CTO-POBA group, whereas those in the CTO-stent and CTO-POBA groups showed no significant differences. After inverse probability of treatment weighting to balance the differences among covariates between the non-CTOstent and non-CTO-POBA groups, the non-CTO-stent group still showed superior clinical primary patency as compared with the non-CTO-POBA group.

Conclusions: In the patients with femoropopliteal stenosis without CTO, stenting resulted in better clinical outcomes than balloon angioplasty. The presence of CTO in the femoropopliteal lesion should be considered when selecting a suitable device for performing endovascular procedures.

Keywords: Peripheral arterial disease; Registry; Balloon angioplasty; Stent; Coronary occlusion 


\section{INTRODUCTION}

Remarkable advances have been made over the last decades in the endovascular treatment of atherosclerotic disease in femoropopliteal arteries. Currently, endovascular treatment of femoropopliteal lesions is the first choice for stenosis/occlusions $<25 \mathrm{~cm}$ [1]. Balloon angioplasty effectively dilates the femoropopliteal lesions without any residues. However, the lack of scaffolds often leads to suboptimal results. Stenting provides a stable initial success with scaffolds while the high rate of stent failure, including stent fracture, thrombosis, and restenosis, is still an unresolved concern on the use of stents in femoropopliteal lesions. Trials have reported contradictory results on the patency of stenting and that of isolated plain old balloon angioplasty (POBA) for femoropopliteal occlusive disease. A meta-analysis reported that stent placement in femoropopliteal occlusive disease does not increase the patency rate when compared with angioplasty alone at 1 year [2]. Conversely, primary stenting with self-expanding nitinol stents for the treatment of superficial femoral artery (SFA) obstructions yields a sustained morphological benefit and a trend toward clinical benefit compared with balloon angioplasty with optional stenting [3]. In a single-center retrospective registry, stenting resulted in similar long-term outcomes as those of POBA when stratified between claudication and critical limb ischemia [4]. However, stenting yielded a statistically better primary patency only in the patients with TransAtlantic Inter-Society Consensus classification (TASC-II) C or D lesions. Therefore, whether stenting results in a therapeutic benefit as compared with balloon angioplasty remains controversial.

In the present study, we analyzed data from a multicenter cohort of patients with lower-extremity peripheral arterial disease (PAD) who underwent endovascular treatment. We determined the effects of POBA in comparison with those of stenting on patency for the treatment of femoropopliteal in the presence or absence of chronic total occlusion (CTO).

\section{METHODS}

\section{Study population}

The Korean Vascular Intervention Society Endovascular
Therapy in Lower-Limb Artery Diseases (K-VIS ELLA) registry is a multicenter, observational, retrospective, and prospective study of patients with lower extremity artery diseases treated with endovascular therapy (ClinicalTrials.gov NCTo2748226). The present study used data from the retrospective patient cohort, which consists of datasets from 3,073 patients with 3,972 target limbs treated between January 2006 and July 2015 in 31 Korean hospitals [5]. Data regarding the patient baseline clinical, lesion, and procedural characteristics were collected from their electronic medical records. We excluded patients who did not receive femoropopliteal intervention (Fig. 1). We also excluded patients who were treated with drug-coated balloon or drug-eluting stents. We divided the patients into 4 groups according to the presence of CTO and use of stenting as follows: CTO-POBA, patients with CTO treated with POBA; CTO-stent, patients with CTO treated with stenting; non-CTO-POBA, patients without CTO treated with POBA; non-CTO-stent, patients without CTO treated with stenting. The study was conducted in accordance with the principles of the Declaration of Helsinki and approved by the Institutional Review Boards of the participating hospitals (Seoul National University Bundang Hospital, B-1603/340-304). The Institutional Review Boards of the participating hospitals waived the requirement for informed consent for this retrospective study.

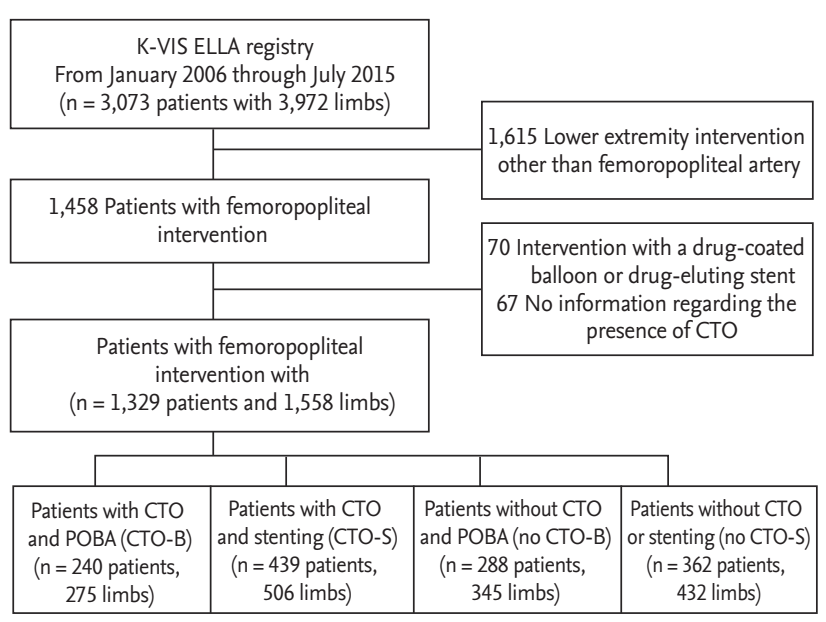

Figure 1. Study flow. K-VIS ELLA, the Korean Vascular Intervention Society Endovascular Therapy in Lower-Limb Artery Diseases; CTO, chronic total occlusion; POBA, plain old balloon angioplasty. 


\section{Definitions}

PAD of the lower extremity was defined as the presence of $\geq 50 \%$ narrowing of a lower-extremity artery. Claudication was defined as Rutherford category 1, 2, or 3 disease (mild, moderate, or severe claudication, respectively), and critical limb ischemia was defined as Rutherford category 4, 5, or 6 disease (ischemic rest pain, minor tissue loss, or major tissue loss, respectively) [6]. The presence of hypercholesterolemia was defined as a total cholesterol level of $>200 \mathrm{mg} / \mathrm{dL}$ or treatment with a lipid-lowering agent prior to hospital admission, as documented in the medical records. Patients were considered current smokers if they smoked at least one cigarette per day within the last month. Patients were considered ex-smokers if they had stopped smoking at least 1 month prior to inclusion in the study. Congestive heart failure was defined as the presence of a left ventricular ejection fraction $<40 \%$. Chronic kidney disease (CKD) was considered to be present if the estimated glomerular filtration rate according to the Modification of Diet in Renal Disease Study equation was $<60 \mathrm{~mL} /$ $\min / 1.73 \mathrm{~m}^{2}$.

Target vessels were classified as aortoiliac, femoropopliteal, anterior tibial, posterior tibial, and peroneal arteries. Target lesions of the femoropopliteal arteries were classified according to the TASC-II classification [7]. Multilevel disease was defined as the presence of significant obstructive lesions at $>1$ level in the same limb (aortoiliac, femoropopliteal, and infrapopliteal). The prescribed antiplatelet drugs included aspirin, clopidogrel, ticlopidine, ticagrelor, prasugrel, and cilostazol.

\section{Endovascular procedure}

The crossover or antegrade approach was selected as appropriate. Intraluminal or subintimal wire crossing was achieved using 0.014 - or 0.035 -in guidewires at the discretion of the operators. Balloon angioplasty with a standard balloon was performed in both groups, and the maximal diameter of the balloon was recorded. All the patients in the stent groups received nitinol stents, including Smart Stent (Cordis, Johnson \& Johnson, Fremont, CA, USA), Complete SE (Medtronic Inc., Minneapolis, MN, USA), or Absolute pro (Abbott, Santa Clara, CA, USA). The sum of the stent length and the maximal diameter of the stents were recorded. Technical success was defined as a residual stenosis of $<30 \%$ by final an- giography. Clinical success was defined as improvement in $>1$ categories in the Rutherford class after the procedure.

\section{Patient and lesion follow-up}

The patients and target lesions were followed up postoperatively at 6,12 , and 24 months. We examined the results of the patient's ankle-brachial index (ABI), duplex ultrasonography, computed tomographic angiography, and conventional peripheral angiography, which were performed at the physician's discretion during the follow-up period.

\section{End points}

The primary end point was clinical primary patency, defined as a composite of freedom from an ABI reduction of $\geq 20 \%$ or $>0.15$ in comparison with the post-intervention $A B I,>50 \%$ restenosis, as determined by duplex ultrasound (peak systolic velocity ratio < 2.4), computed tomographic angiography, or digital subtraction angiography, and freedom from clinically driven target lesion revascularization (TLR). Clinically driven TLR was adjudicated when the physicians considered reinterventions at the target lesion or if the patient complained of exertional limb discomfort or claudication within the follow-up period.

The secondary end point consisted of safety end points, including the amputation and mortality rates. We classified amputations performed above the ankle as major amputations. Amputations performed below the ankle that did not require the use of prosthesis and did not affect walking were defined as minor amputations $[8,9]$. Major bleeding was defined as bleeding that caused a decrease in hemoglobin level of $\geq 20 \mathrm{~g} / \mathrm{L}(1.24 \mathrm{mmol} / \mathrm{L})$ or that led to transfusion of $\geq 2$ units of whole blood or red cells [10].

\section{Statistical methods}

Continuous variables were expressed as means with standard deviations, whereas categorical variables were presented as numbers with percentages. Continuous variables were compared using the Student $t$ test. Categorical variables were compared using the chi-square test. Survival times were censored at the date of the end point or last follow-up. The Kaplan-Meier product-limit estimator and the log-rank test were used to analyze 
continuous survival times, and the mixed-effects Cox proportional hazards regression modeling was used to test the interaction of treatment assignment and subgroup factors, as well as multivariate modeling of risk factors. Covariates for the adjustment were selected using the stepwise Akaike information criterion (AIC) method. We included as covariates all the variables found to be statistically significant $(p<0.05)$ in the univariate analysis of the multivariable analysis or variables known to be clinically important, excluding those with multicollinearity with others. The proportional hazard assumption of each variable was tested on the basis of Schoenfeld residuals. The model included the available patient characteristics, clinical risk factors, lesion characteristics, and procedural data. To minimize bias by indication and missing values, an inverse-probability treatment-weighted(IPTW) cohort was created using the "twang package" after multiple imputation of missing values by the "MICE package" in the R program (The R Foundation for Statistical Computing, Vienna, Austria; http://www.R-project.org). We provided a pooled result from 5 different datasets by multiple imputation. A two-sided $p$ value of $<0.05$ was considered indicative of a statistically significant difference. Statistical analyses were performed using R programming version 3.4.2 (The R Foundation for Statistical Computing). All the statistical analyses were performed by a professional statistician (S.H.K.).

\section{RESULTS}

\section{Patient and lesion characteristics}

The baseline demographic and clinical characteristics of

Table 1. Patients' baseline characteristics according to the presence of CTO

\begin{tabular}{|c|c|c|c|c|c|c|}
\hline \multirow[b]{2}{*}{ Characteristic } & \multicolumn{3}{|c|}{ Non-CTO $(\mathrm{n}=781)$} & \multicolumn{3}{|c|}{ CTO (n = 674) } \\
\hline & $\begin{array}{c}\text { POBA } \\
(n=288)\end{array}$ & $\begin{array}{l}\text { Stenting } \\
(\mathrm{n}=362)\end{array}$ & $p$ value & $\begin{array}{c}\text { POBA } \\
(n=240)\end{array}$ & $\begin{array}{l}\text { Stenting } \\
(\mathrm{n}=439)\end{array}$ & $p$ value \\
\hline Age, yr & $68.34 \pm 8.34$ & $69.48 \pm 9.48$ & 0.098 & $69.08 \pm 9.080$ & $69.94 \pm 9.94$ & 0.276 \\
\hline Male sex & $217(75 \cdot 3)$ & $287(79 \cdot 3)$ & 0.272 & $175(72.9)$ & $369(84.1)$ & 0.001 \\
\hline $\mathrm{BMI}, \mathrm{kg} / \mathrm{m}^{2}$ & $23.95 \pm 3.95$ & $23.54 \pm 3.54$ & 0.176 & $23.61 \pm 3.61$ & $23.07 \pm 3.07$ & 0.083 \\
\hline Hypertension & $242(84.0)$ & $287(79 \cdot 3)$ & 0.149 & $172(71.7)$ & $319(72.7)$ & 0.851 \\
\hline $\mathrm{DM}$ & $224(77.8)$ & $245(67 \cdot 7)$ & 0.006 & $140(58.3)$ & $219(49 \cdot 9)$ & 0.043 \\
\hline Hypercholesterolemia & $110(38.2)$ & $172(47 \cdot 5)$ & 0.021 & $99(41.2)$ & $158(36.0)$ & 0.205 \\
\hline Current smoker & $54(18.8)$ & $96(26.5)$ & 0.025 & $67(27.9)$ & $172(39.2)$ & 0.004 \\
\hline $\mathrm{CHF}$ & $21(7 \cdot 3)$ & $24(6.6)$ & 0.861 & $19(7 \cdot 9)$ & $33(7 \cdot 5)$ & 0.971 \\
\hline CAD & $185(64.2)$ & $216(59 \cdot 7)$ & 0.268 & $128(53 \cdot 3)$ & $238(54.2)$ & 0.889 \\
\hline CKD & $106(36.8)$ & $84(23.2)$ & $<0.001$ & $51(21.2)$ & $66(15 \cdot 0)$ & 0.052 \\
\hline Previous stroke & $57(19.8)$ & $50(13.8)$ & 0.053 & $37(15 \cdot 4)$ & $67(15 \cdot 3)$ & $>0.999$ \\
\hline Previous bypass surgery & $41(14.2)$ & $33(9.1)$ & 0.055 & $15(6.2)$ & $28(6.4)$ & $>0.999$ \\
\hline Previous amputation & $40(13.9)$ & $14(3 \cdot 9)$ & $<0.001$ & $20(8.3)$ & $18(4 \cdot 1)$ & 0.034 \\
\hline Previous PTA & $50(17 \cdot 4)$ & $47(13.0)$ & 0.148 & $33(13.8)$ & $50(11.4)$ & 0.438 \\
\hline CLI & $131(45 \cdot 5)$ & $102(28.2)$ & $<0.001$ & $102(42.5)$ & $136(31.0)$ & 0.003 \\
\hline Aspirin & $242(86.7)$ & $312(87.2)$ & 0.972 & $207(87.7)$ & $375(87 \cdot 4)$ & $>0.999$ \\
\hline Clopidogrel & $231(82.8)$ & $318(88.8)$ & 0.038 & $182(77.1)$ & $371(86.5)$ & 0.003 \\
\hline Cilostazol & $103(36.9)$ & $137(38.3)$ & 0.790 & $109(46.2)$ & $198(46.2)$ & $>0.999$ \\
\hline Statin & $196(70.3)$ & $275(76.8)$ & 0.075 & $165(69.9)$ & $299(69.7)$ & $>0.999$ \\
\hline
\end{tabular}

Values are presented as mean \pm SD or number $(\%)$.

CTO, chronic total occlusion; POBA, plain old balloon angioplasty; BMI, body mass index; DM, diabetes mellitus; CHF, congestive heart failure; CAD, coronary artery disease; CKD, chronic kidney disease; PTA, percutaneous transluminal angioplasty; CLI, critical limb ischemia. 
the patients in the four groups are summarized in Table 1. Baseline patient sex and preexisting risk factors (body mass index, diabetes, smoking status, and CKD) were significantly different between the groups. In addition, the incidence of critical limb ischemia and previous amputation were significantly different between the groups. Clopidogrel was prescribed at a significantlyhigher rate in the stenting group.

Among 1,558 limbs, the numbers of limbs in the nonCTO-POBA, non-CTO-stent, CTO-POBA, and CTOstent were 345, 432, 275, and 506, respectively (Table 2). The number of patients with aortoiliac lesion was significantly higher in the stent group, whereas that of patients with infrapopliteal lesions was higher in the POBA group. Lesion characteristics differed between the two treatment groups. The mean lesion length was longer in the stenting and CTO groups. The maximal balloon diameter was significantly larger in the stenting group. The maximal stent diameter was larger and the mean stented length was shorter in the non-CTO- stent group than in the CTO-stent group. Technical and clinical successes and good antegrade flow were more frequently achieved in the stenting group. The postprocedural target limb ABI did not differ between the two groups.

\section{Postprocedural follow-up}

The median follow-up duration was 509 days (interquartile range, 246 to 721 ). The clinical primary patency in the non-CTO-stent group was significantly higher than that in the non-CTO-POBA group $(p=0.041)$, whereas it did not differ between the CTO-stent and CTO-POBA groups $(p=0.670)$ (Fig. 2A). Freedom from TLR was also higher in the non-CTO-stent group than in the nonCTO-POBA group but showed no significant difference between the CTO-POBA and CTO-stent groups (Fig. 2B). The estimated clinical primary patency and TLR rates are summarized in Supplementary Table 1. Evaluation of either the whole population or patients divided according to TASC-II classifications (TASC-II A and B vs. C and

Table 2. Lesion and procedural characteristics according to the presence of CTO

\begin{tabular}{|c|c|c|c|c|c|c|}
\hline \multirow{2}{*}{ Characteristic } & \multicolumn{3}{|c|}{ Non-CTO } & \multicolumn{3}{|c|}{ CTO } \\
\hline & $\operatorname{POBA}(\mathrm{n}=345)$ & Stenting $(n=432)$ & $p$ value & $\operatorname{POBA}(n=275)$ & Stenting $(n=506)$ & $p$ value \\
\hline ABI target & $0.69 \pm 0.24$ & $0.64 \pm 0.19$ & 0.019 & $0.58 \pm 0.24$ & $0.50 \pm 0.20$ & $<0.001$ \\
\hline ABI nontarget & $0.78 \pm 0.24$ & $0.78 \pm 0.23$ & 0.780 & $0.84 \pm 0.27$ & $0.74 \pm 0.23$ & 0.001 \\
\hline Aortoiliac lesion & $49(14.2)$ & $115(26.6)$ & $<0.001$ & $29(10.5)$ & $157(31.0)$ & $<0.001$ \\
\hline Below-the-knee lesion & $202(58.6)$ & $130(30.1)$ & $<0.001$ & $126(45.8)$ & $173(34.2)$ & 0.002 \\
\hline Lesion length, mm & $127.97 \pm 105.80$ & $136.02 \pm 106.23$ & 0.318 & $159.70 \pm 103.38$ & $198.94 \pm 108.86$ & $<0.001$ \\
\hline Maximal balloon diameter, $\mathrm{mm}$ & $5.28 \pm 1.22$ & $5.51 \pm 2.06$ & 0.057 & $5.20 \pm 0.96$ & $5.68 \pm 2.82$ & 0.001 \\
\hline Balloon length, mm & $166.75 \pm 147.79$ & $117.16 \pm 107.55$ & $<0.001$ & $156.65 \pm 130.29$ & $141.59 \pm 110.34$ & 0.134 \\
\hline Max stent diameter, mm & & $6.97 \pm 4.25$ & & & $6.75 \pm 0.84$ & \\
\hline Stent length sum, mm & & $102.94 \pm 59.17$ & & & $152.62 \pm 85.11$ & \\
\hline Technical success & & & $<0.001$ & & & $<0.001$ \\
\hline No & $3(0.9)$ & $10(2.4)$ & & $26(9.6)$ & $14(2.8)$ & \\
\hline Suboptimal & $32(9 \cdot 4)$ & $6(1.4)$ & & $30(11.1)$ & $21(4.2)$ & \\
\hline Yes & $304(89.7)$ & $409(96.2)$ & & $214(79 \cdot 3)$ & $466(93.0)$ & \\
\hline Good antegrade flow & $334(98.5)$ & $413(97 \cdot 2)$ & 0.313 & $239(88.5)$ & $477(95.2)$ & 0.001 \\
\hline Residual stenosis, $<30 \%$ & $305(90.0)$ & $415(97 \cdot 6)$ & $<0.001$ & $216(80.0)$ & $472(94.2)$ & $<0.001$ \\
\hline Contrast volume, $\mathrm{mL}$ & $156.26 \pm 78.84$ & $159.05 \pm 97.70$ & 0.702 & $183.38 \pm 88.95$ & $185 \cdot 30 \pm 3 \cdot 5^{2}$ & 0.814 \\
\hline Clinical success & $197(57.1)$ & $323(74.8)$ & $<0.001$ & $139(50.5)$ & $340(67.2)$ & $<0.001$ \\
\hline Post-ABI & $0.82 \pm 0.22$ & $0.87 \pm 0.18$ & 0.038 & $0.84 \pm 0.25$ & $0.84 \pm 0.19$ & 0.836 \\
\hline
\end{tabular}

Values are presented as mean \pm SD or number (\%).

CTO, chronic total occlusion; POBA, plain old balloon angioplasty; ABI, ankle-brachial index. 

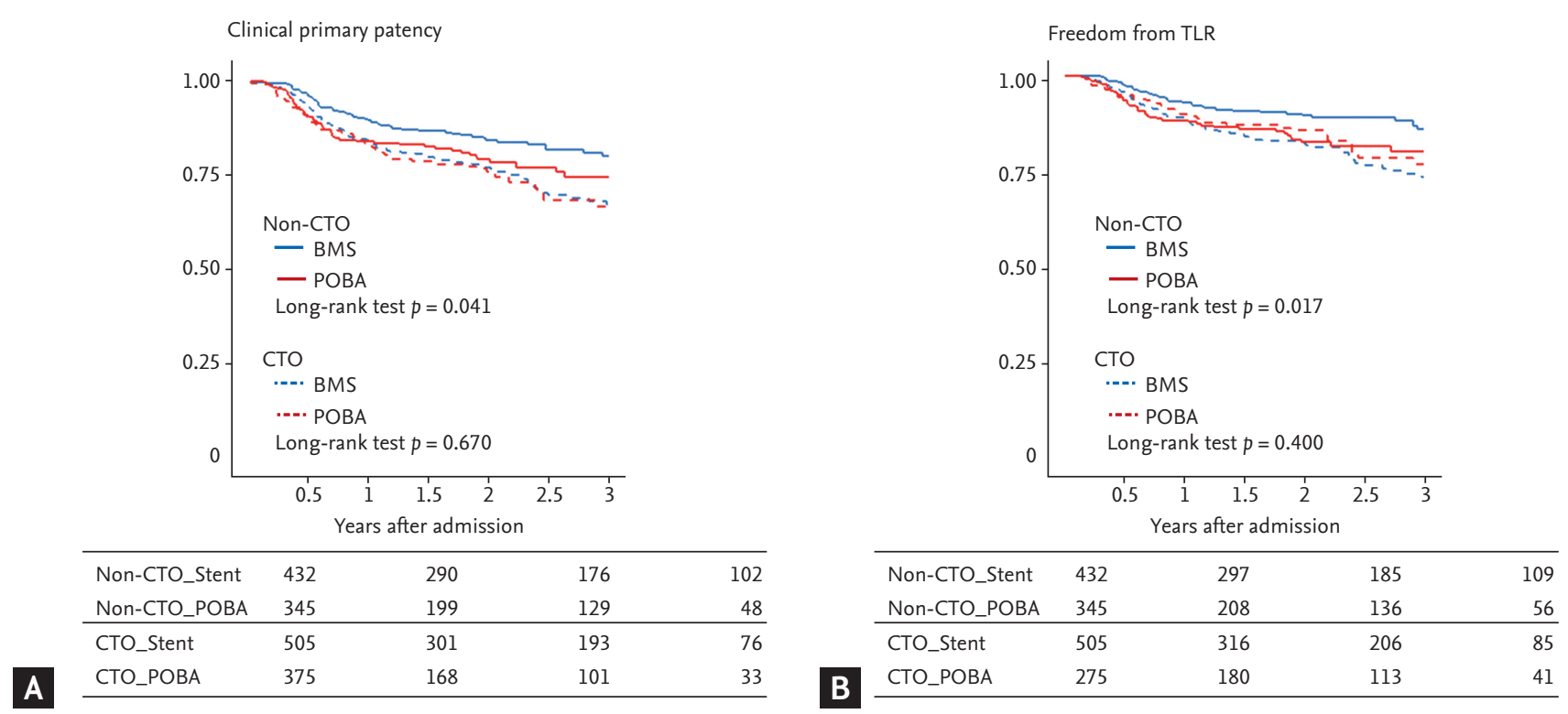

Figure 2. Kaplan-Meier curves for outcomes. Kaplan-Meier curves for clinical primary patency and target vessel revascularization in non-the chronic total occlusion (CTO) and CTO groups. (A) Clinical primary patency, (B) freedom from target lesion revascularization (TLR). BMS, bare metal stent; POBA, plain old balloon angioplasty.

D) did not result in any difference in outcome between stenting or the POBA procedures (Supplementary Tables 2-5, Supplementary Figs. 1 and 2).

We performed a Cox proportional hazard analysis to identify and adjust covariates, which may have affected the clinical primary patency rate. Hypercholesterolemia and lesion length were significantly associated with the clinical primary patency rate in both groups, whereas chronic renal failure was differentially associated with the outcome of CTO and non-CTO lesions. Even after adjustment with the covariates, stenting was associated with a better clinical primary patency than balloon angioplasty only in the non-CTO lesion group (Table 3).

With regard to the unbalanced baseline characteristics between the POBA and stenting groups, we performed IPTW analyses to test a causal inference from our retrospective data. Most covariates were well balanced after IPTW except for chronic renal failure, critical limb ischemia, and below-the-knee lesion in the non-CTO group (Table 4). We found that stenting in the nonCTO group led to a better clinical primary patency and freedom from TLR than did the POBA treatment in the non-CTO group in the IPTW analysis $(p=0.05$ and $p=$ ..034, respectively) and in the adjustments for chronic renal failure, critical limb ischemia, and below-the-knee lesion (hazard ratio [HR], 1.43 [1.08 to 1.91]; $p=0.014 ; \mathrm{HR}$, 1.61 [1.15 to 2.26]; $p=0.006$, respectively) (Fig. 3). However, in the CTO group, no significant difference was found between the treatment groups.

\section{Sensitivity analyses}

Several sensitivity analyses were performed to verify the robustness of the results using exploratory subgroups, including (1) de novo lesion only after the exclusion of a previously treated lesion, (2) technical success only after the exclusion of technical failure, and (3) good antegrade flow after the procedure. In any subgroup analysis, stenting in the non-CTO group led to a better clinical primary patency and freedom from TLR than did POBA in the non-CTO group (Supplementary Figs. 3-5).

\section{DISCUSSION}

In the present study, we determined for the first time the effects of POBA in comparison with those of stenting on clinical primary patency in patients with femoropopliteal CTO and non-CTO lesions. The nonCTO-stent group showed a significantly better clinical primary patency than the no-CTO-POBA group, where- 


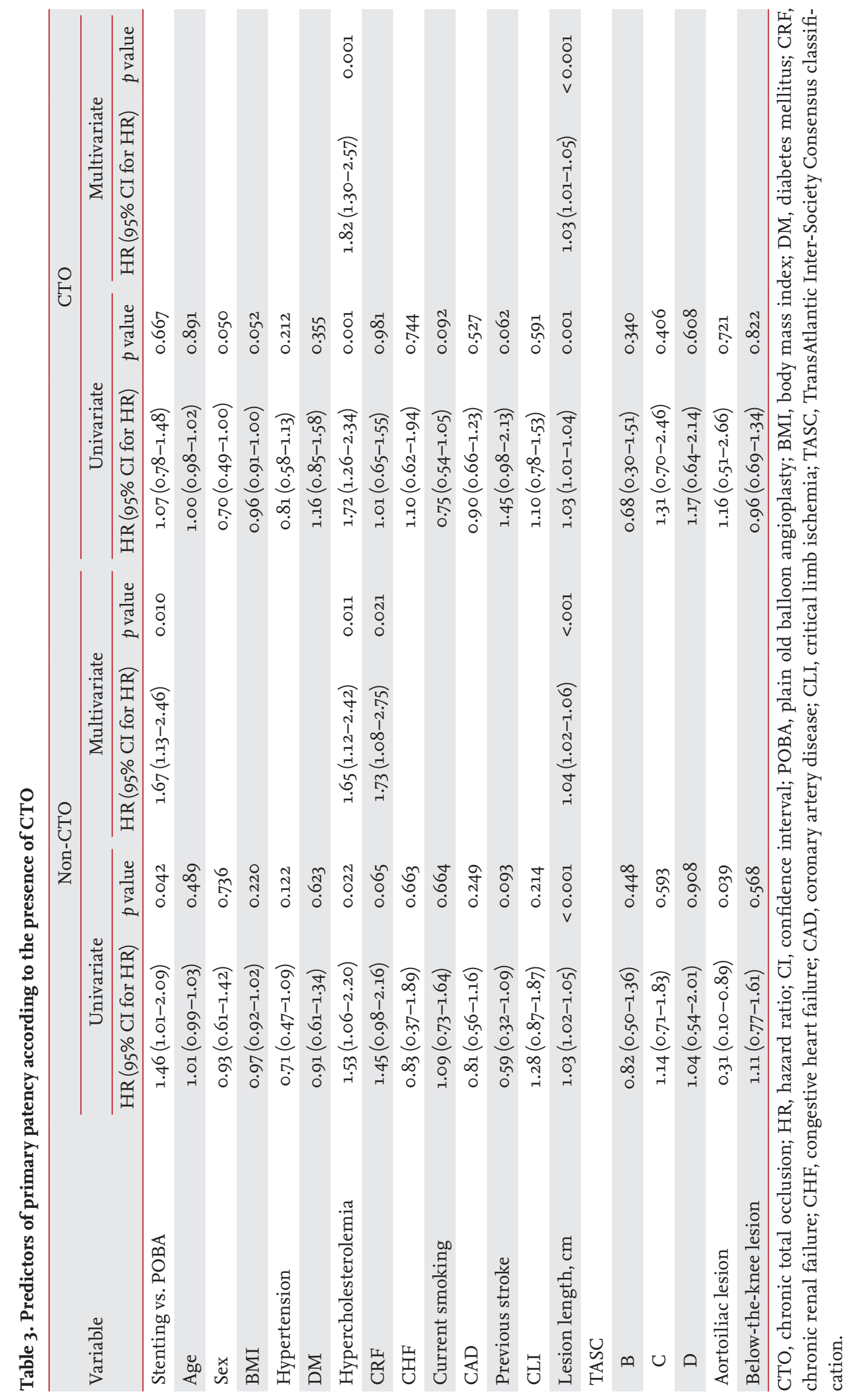


Table 4. Characteristics according to the presence of CTO after inverse probability treatment weighting

\begin{tabular}{|c|c|c|c|c|c|c|c|c|}
\hline \multirow{2}{*}{ Characteristic } & \multicolumn{4}{|c|}{ Non-CTO } & \multicolumn{4}{|c|}{ CTO } \\
\hline & Stenting & POBA & SMD & $p$ value & Stenting & POBA & SMD & $p$ value \\
\hline Age, yr & $69.77 \pm 8.52$ & $69.39 \pm 8.78$ & 0.044 & 0.850 & $69.81 \pm 9.45$ & $69.57 \pm 9.72$ & 0.026 & 0.946 \\
\hline Male sex & $502(76.83)$ & $473(76.13)$ & 0.017 & 0.829 & $565(82.39)$ & $462(77.57)$ & 0.127 & 0.118 \\
\hline BMI, kg/m² & $23.64 \pm 3.76$ & $23.73 \pm 3.60$ & 0.022 & 0.726 & $23.26 \pm 3.73$ & $23.37 \pm 3.93$ & 0.029 & 0.874 \\
\hline HTN & $308(47.21)$ & $262(42.25)$ & 0.099 & 0.197 & $261(38.03)$ & $251(42.14)$ & 0.085 & 0.317 \\
\hline $\mathrm{DM}$ & $459(70.32)$ & $461(74.26)$ & 0.086 & 0.268 & $351(51.18)$ & $327(54.85)$ & 0.073 & 0.387 \\
\hline Hypercholesterolemia & $308(47.21)$ & $262(42.25)$ & 0.099 & 0.197 & $261(38.03)$ & $251(42.14)$ & 0.085 & 0.317 \\
\hline $\mathrm{CRF}$ & $162(24.80)$ & $202(32.46)$ & 0.177 & 0.028 & $109(15.87)$ & $95(16.00)$ & 0.004 & 0.963 \\
\hline $\mathrm{CHF}$ & $46(6.97)$ & $45(7.24)$ & 0.011 & 0.892 & $52(7 \cdot 56)$ & $37(6.19)$ & 0.052 & 0.470 \\
\hline Smoking & $172(26.32)$ & $121(19.43)$ & 0.157 & 0.180 & $267(38.97)$ & $206(34.55)$ & 0.091 & 0.293 \\
\hline CAD & $386(59.11)$ & $407(65.51)$ & 0.130 & 0.089 & $377(54.98)$ & $338(56.72)$ & 0.035 & 0.675 \\
\hline Stroke & $91(13.98)$ & $110(17.75)$ & 0.109 & 0.180 & $112(16.38)$ & $88(14.80)$ & 0.043 & 0.611 \\
\hline CLI & $187(28.66)$ & $225(36.24)$ & 0.168 & 0.034 & $221(32.31)$ & 189 (31.69) & 0.013 & 0.869 \\
\hline Lesion length, cm & $13.13 \pm 10.21$ & $12.94 \pm 10.41$ & 0.019 & 0.482 & $19.09 \pm 11.00$ & $17.45 \pm 10.86$ & 0.149 & 0.249 \\
\hline TASC & & & & 0.516 & & & & 0.774 \\
\hline A & $150(22.97)$ & $136(21.84)$ & 0.027 & & $58(8.51)$ & $48(8.01)$ & 0.018 & \\
\hline $\mathrm{B}$ & $206(31.57)$ & $221(35.56)$ & 0.086 & & $77(11.26)$ & $76(12.78)$ & 0.048 & \\
\hline $\mathrm{C}$ & $224(34.23)$ & $185(29.80)$ & 0.094 & & $161(23.46)$ & $156(26.15)$ & 0.063 & \\
\hline $\mathrm{D}$ & $73(11.22)$ & $79(12.80)$ & 0.050 & & $389(56.77)$ & $316(53.06)$ & 0.075 & \\
\hline Aortoiliac lesion & $0.64 \pm 0.21$ & $0.68 \pm 0.23$ & 0.176 & 0.169 & $0.50 \pm 0.22$ & $0.55 \pm 0.22$ & 0.212 & 0.260 \\
\hline Below-the-knee lesion & $239(36.61)$ & $302(48.62)$ & 0.249 & 0.002 & $242(35 \cdot 35)$ & $256(42.89)$ & 0.158 & 0.065 \\
\hline
\end{tabular}

Values are presented as mean \pm SD or number (\%). Inverse probability treatment weighting with age, sex, BMI, DM, chronic kidney disease, previous amputation, CLI, aortoiliac lesion, below-the-knee lesion, lesion length, maximal balloon diameter. CTO, chronic total occlusion; POBA, plain old balloon angioplasty; SMD, starndardized mean difference; BMI, body mass index; HTN, hypertension; DM, diabetes mellitus; CRF, chronic renal failure; CHF, congestive heart failure; CAD, coronary artery disease; CLI, critical limb ischemia; TASC, TransAtlantic Inter-Society Consensus classification.

as the CTO-stent and CTO-POBA groups showed no significant difference in the crude population. After the IPTW analysis to balance the difference of covariates between the non-CTO-stent and non-CTO-POBA groups, the non-CTO-stent group still showed superior clinical primary patency as compared with the non-CTO-POBA group. Sensitivity analyses also showed robustness of the results in the various subgroups.

\section{Comparison between balloon angioplasty and stenting}

Clinical studies have shown the superiority of stenting to POBA in long SFA lesions [11,12], whereas in the short lesions (< $10 \mathrm{~cm}$ in length) no significant differences were found [13]. A previous meta-analysis showed that a short-term but no long-term benefit of primary patency from the primary stenting of SFA lesions in ad- dition to angioplasty [14]. However, most studies were underpowered to sufficiently discriminate the efficacy of stenting in the CTO and non-CTO groups, and included patients with total occlusions comprising $<30 \%$ of the whole study population. Therefore, we do not have any data regarding the impact of CTO when deciding treatment options for SFA lesions. SFA is subject to longitudinal stretching, external compression, torsion, and flexion, which may lead to stent fractures and eventually restenosis. Therefore, understanding and identifying conditions in which stenting is superior to POBA are important. We demonstrated for the first time that stenting resulted in better clinical primary patency and was inferior to POBA only in the non-CTO lesion group, with a power of $80 \%$. Lesion length was also a significant predictor of clinical primary patency irrespective of the 

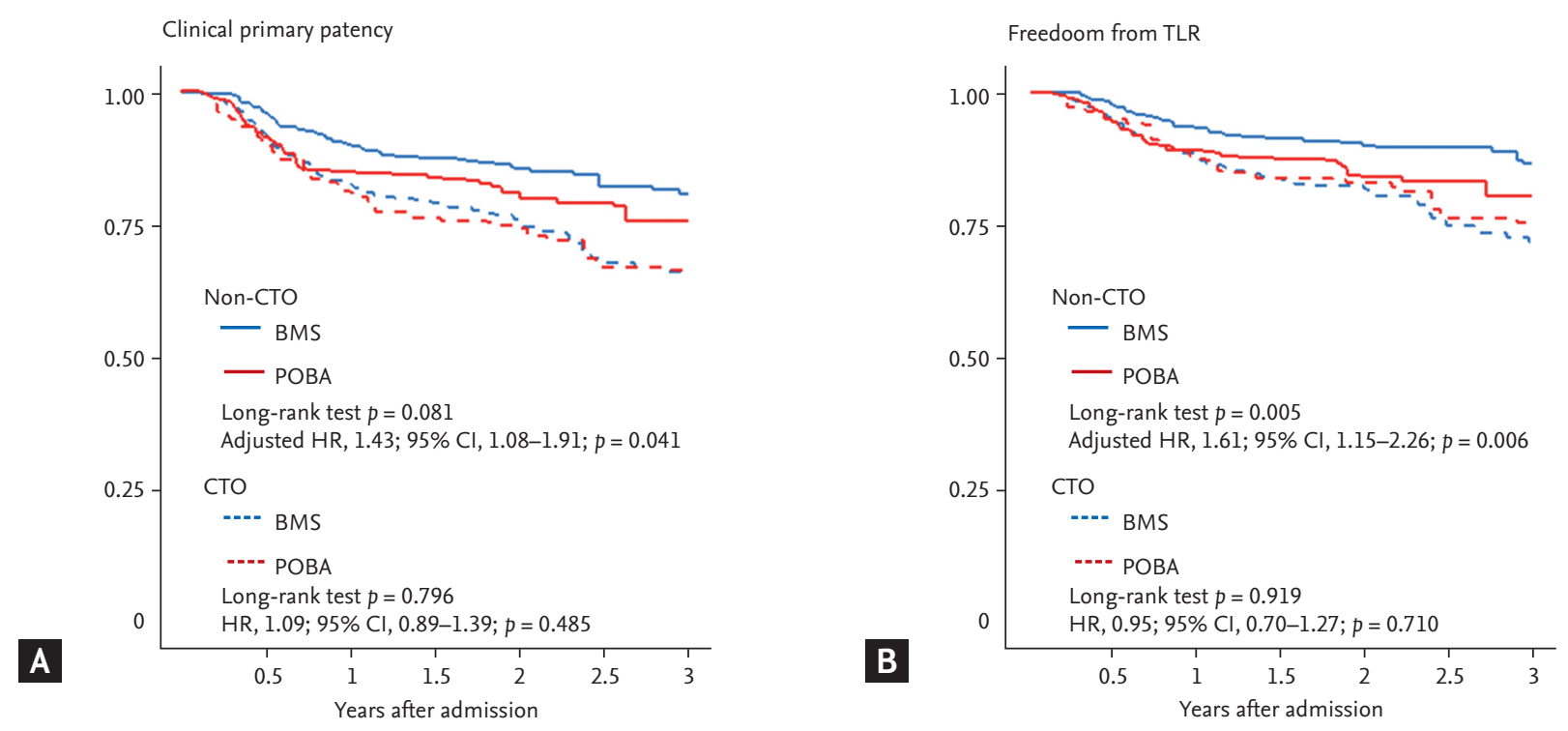

Figure 3. Kaplan-Meier curves for outcomes after inverse probability of treatment weighting. Kaplan-Meier curves for clinical primary patency and target vessel revascularization in the non-chronic total occlusion (CTO) and CTO groups. (A) Clinical primary patency, (B) freedom from target lesion revascularization (TLR). HR, hazard ratio; CI, confidence interval; BMS, bare metal stent; POBA, plain old balloon angioplasty.

presence of CTO in the multivariate Cox proportional hazard analyses. The TASC-II classification is a sophisticated system used to predict patency depending on the lesion length, location, and presence of CTO. However, we found that the TASC-II classification could predict long-term clinical primary patency but could not differentiate any benefit of stenting over POBA for the SFA lesions in our study.

\section{Comparison between CTO and stenosis}

A previous study that investigated the endovascular management of SFA CTO resulted in a reasonable primary patency ranging from $44 \%$ to $58 \%$ and a secondary patency of $92 \%$ [15]. In line with our results, no significant differences were found among the treatment modalities (angioplasty, angioplasty with stenting, and atherectomy). The inability to cross all lesions intraluminally in SFA occlusions may have led to a high rate of subintimal wiring and complications such as perforation or dissection. The procedural complexity in the occluded cases potentially worsened the clinical outcomes in the stenting group, which inevitably included bailout stenting. In a study where stenting was compared with angioplasty only in patients with chronic occlusive disease treated with subintimal angioplasty, 1-year primary patency was not significantly different between the stent and no-stent groups [16].

\section{Stenting or not: lesion or clinical indicators}

In a randomized controlled trial (RCT) that reported the benefit of stenting over balloon angioplasty, the occlusion rate was $31 \%$ in the angioplasty group and $41 \%$ in the stenting group [3]. In our real-world registry, the occlusion rate was $50 \%$, which was significantly higher than in the RCT. The overall stenting efficacy was not different from that of POBA. Therefore, stenting might be recommended only for cases of stenotic SFA lesions. The clinical status may also affect the efficacy of treatment modalities. For example, in CLI patients with SFA CTOs, atherectomy was reported to produce better outcomes than angioplasty alone [15]. The authors also reported that CTOs treated with stenting improved secondary patency rates as compared with those treated with POBA in patients with diabetes. In addition to CTO, CLI, and diabetes mellitus, additional lesion or clinical characteristics need to be identified to warrant the decision of stenting.

\section{Future strategies}

Endovascular therapies superior to POBA or bare-met- 
al stenting have recently attracted considerable interest. Paclitaxel-eluting stents (PES) displayed sustained safety and efficacy compared with POBA in patients with femoral artery disease, with superior clinical efficacy as compared with POBA and provisional stenting [17]. In addition, paclitaxel-coated balloon angioplasty (PEB) for atherosclerotic femoropopliteal disease reduces target-lesion revascularization to a greater degree than POBA, with the advantage of leaving no prosthetic material behind [18-20]. However, decision-making criteria on whether to apply PES or PEB remain to be investigated. Whether PEB angioplasty and stenting may further reduce restenosis and improve long-term clinical outcomes as compared with PEB angioplasty alone in SFA occlusion deserves further investigation. Drug-eluting stents do not seem to be an option for SFA occlusions because a recent registry showed that PES did not improve outcomes as compared with bare-metal stents in TASC-II C and D femoropopliteal lesions [21].

\section{Limitations}

The present data do not represent the results of a randomized controlled study but instead are derived from a multicenter registry. Consequently, risk factors such as diabetes mellitus and critical limb ischemia showed a non-uniform distribution between the groups. Although we adjusted for significant risk factors, unmeasured covariates may have influenced the study outcomes. In our registry, we could not differentiate percutaneous transluminal angioplasty with provisional stenting from primary stenting. As an initial strategy, the 2 treatment options have different implications for operators in terms of procedure planning and may lead to different clinical outcomes. Our results reflect the clinical outcomes of the endovascular procedure ultimately chosen by the operators, although it is difficult to separate POBA from stenting because the femoropopliteal lesion is usually very long and treated with mixed methods.

In conclusion, in patients with femoropopliteal stenosis without CTO, stenting demonstrated better clinical outcomes than balloon angioplasty. The presence of CTO in femoropopliteal lesions should be considered when selecting a device to be used in endovascular procedures.

\section{KEY MESSAGE}

1. Trials have reported contradictory results on the patency of stenting and that of isolated plain old balloon angioplasty for femoropopliteal occlusive disease. Moreover, whether the presence of chronic total occlusion (CTO) affects the patency after stenting in cases of femoropopliteal lesion is unknown.

2. We found that stenting demonstrated better clinical outcomes than did balloon angioplasty in patients with femoropopliteal stenosis without CTO.

3. Future randomized controlled trials are necessary to confirm these results.

\section{Conflict of interest}

No potential conflict of interest relevant to this article was reported.

\section{Acknowledgments}

The authors gratefully acknowledge the active participation of all the K-VIS investigators in this study.

This research was supported by a grant from the Korean Health Technology R\&D Project, Ministry of Health and Welfare, Republic of Korea (HI19Co655); and a grant from the SNUBH Research Fund (16-2017-003).

\section{REFERENCES}

1. Aboyans V, Ricco JB, Bartelink MEL, et al. 2017 ESC guidelines on the diagnosis and treatment of peripheral arterial diseases, in collaboration with the European Society for Vascular Surgery (ESVS): Document covering atherosclerotic disease of extracranial carotid and vertebral, mesenteric, renal, upper and lower extremity arteriesEndorsed by: the European Stroke Organization (ESO) The Task Force for the Diagnosis and Treatment of Peripheral Arterial Diseases of the European Society of Cardiology (ESC) and of the European Society for Vascular Surgery (ESVS). Eur Heart J 2018;39:763-816.

2. Mwipatayi BP, Hockings A, Hofmann M, Garbowski M, Sieunarine K. Balloon angioplasty compared with stenting for treatment of femoropopliteal occlusive disease: a 
meta-analysis. J Vasc Surg 2008;47:461-469.

3. Schillinger M, Sabeti S, Dick P, et al. Sustained benefit at 2 years of primary femoropopliteal stenting compared with balloon angioplasty with optional stenting. Circulation 2007;115:2745-2749.

4. Nguyen BN, Conrad MF, Guest JM, et al. Late outcomes of balloon angioplasty and angioplasty with selective stenting for superficial femoral-popliteal disease are equivalent. J Vasc Surg 2011;54:1051-1057.

5. Ko YG, Ahn CM, Min PK, et al. Baseline characteristics of a retrospective patient cohort in the Korean vascular intervention society endovascular therapy in lower limb artery Diseases (K-VIS ELLA) Registry. Korean Circ J 2017;47:469-476.

6. Rutherford RB, Baker JD, Ernst C, et al. Recommended standards for reports dealing with lower extremity ischemia: revised version. J Vasc Surg 1997;26:517-538.

7. Norgren L, Hiatt WR, Dormandy JA, et al. Inter-society consensus for the management of peripheral arterial disease (TASC II). J Vasc Surg 2007;45 Suppl S:S5-S67.

8. Dos Santos VP, da Silveira DR, Caffaro RA. Risk factors for primary major amputation in diabetic patients. Sao Paulo Med J 2006;124:66-70.

9. Wang C, Mai L, Yang C, et al. Reducing major lower extremity amputations after the introduction of a multidisciplinary team in patient with diabetes foot ulcer. BMC Endocr Disord 2016;16:38.

10. Cutlip DE, Windecker S, Mehran R, et al. Clinical end points in coronary stent trials: a case for standardized definitions. Circulation 2007;115:2344-2351.

11. Schillinger M, Sabeti S, Loewe C, et al. Balloon angioplasty versus implantation of nitinol stents in the superficial femoral artery. N Engl J Med 2006;354:1879-1888.

12. Laird JR, Katzen BT, Scheinert D, et al. Nitinol stent implantation versus balloon angioplasty for lesions in the superficial femoral artery and proximal popliteal artery: twelve-month results from the RESILIENT randomized trial. Circ Cardiovasc Interv 2010;3:267-276.
13. Krankenberg H, Schluter M, Steinkamp HJ, et al. Nitinol stent implantation versus percutaneous transluminal angioplasty in superficial femoral artery lesions up to $10 \mathrm{~cm}$ in length: the femoral artery stenting trial (FAST). Circulation 2007;116:285-292.

14. Chowdhury MM, McLain AD, Twine CP. Angioplasty versus bare metal stenting for superficial femoral artery lesions. Cochrane Database Syst Rev 2014;6:CDoo6767.

15. Gallagher KA, Meltzer AJ, Ravin RA, et al. Endovascular management as first therapy for chronic total occlusion of the lower extremity arteries: comparison of balloon angioplasty, stenting, and directional atherectomy. J Endovasc Ther 2011;18:624-637.

16. Schmieder GC, Richardson AI, Scott EC, Stokes GK, Meier GH 3rd, Panneton JM. Selective stenting in subintimal angioplasty: analysis of primary stent outcomes. J Vasc Surg 2008;48:1175-118o.

17. Zeller T, Dake MD, Tepe G, et al. Treatment of femoropopliteal in-stent restenosis with paclitaxel-eluting stents. JACC Cardiovasc Interv 2013;6:274-281.

18. Werk M, Langner S, Reinkensmeier B, et al. Inhibition of restenosis in femoropopliteal arteries: paclitaxel-coated versus uncoated balloon: femoral paclitaxel randomized pilot trial. Circulation 2008;118:1358-1365.

19. Liistro F, Grotti S, Porto I, et al. Drug-eluting balloon in peripheral intervention for the superficial femoral artery: the DEBATE-SFA randomized trial (drug eluting balloon in peripheral intervention for the superficial femoral artery). JACC Cardiovasc Interv 2013;6:1295-1302.

20. Tepe G, Laird J, Schneider P, et al. Drug-coated balloon versus standard percutaneous transluminal angioplasty for the treatment of superficial femoral and popliteal peripheral artery disease: 12-month results from the IN.PACT SFA randomized trial. Circulation 2015;131:495-502.

21. Vent PA, Kaladji A, Davaine JM, et al. Bare metal versus paclitaxel-eluting stents for long femoropopliteal lesions: prospective cohorts comparison using a propensity score-matched analysis. Ann Vasc Surg 2017;43:166-175. 
Supplementary Table 1. Estimated clinical primary patency and target lesion revascularization rate with $95 \%$ CI

\begin{tabular}{|c|c|c|c|c|}
\hline Variable & Non-CTO-POBA & Non-CTO-Stenting & СТО-РОВА & CTO-Stenting \\
\hline \multicolumn{5}{|c|}{ Estimated clinical primary patency rate with 95\% CI (\%) } \\
\hline $1 \mathrm{yr}$ & $84.2(80.1-88.5)$ & $89.6(86.6-92.8)$ & $83.7(79.2-88.5)$ & $83.9(80.5-87.5)$ \\
\hline $2 \mathrm{yr}$ & $79 \cdot 5(74.7-84.6)$ & $84.5(80.7-88.6)$ & $76.1(70.5-82.1)$ & $77.1(72.9-81.5)$ \\
\hline $3 \mathrm{yr}$ & $74.7(68.4-81.5)$ & $80.4(75.6-85.5)$ & $66.8(58.7-76.1)$ & $67 \cdot 4(61.6-73 \cdot 7)$ \\
\hline $4 \mathrm{yr}$ & $69.3(61.4-78.3)$ & $77.7(72.2-83.6)$ & $64.2(55.2-74.7)$ & $62.7(55.9-70.4)$ \\
\hline $5 \mathrm{yr}$ & $65.0(55.8-75.6)$ & $71.2(64.1-79.2)$ & $64.2(55.2-74.7)$ & $61.3(54.2-69.3)$ \\
\hline \multicolumn{5}{|c|}{ Estimated target lesion revascularization rate with 95\% CI (\%) } \\
\hline $1 \mathrm{yr}$ & $88.5(84.8-92.3)$ & $93.0(90.4-95.7)$ & $89.9(86.2-93.9)$ & $89.0(86.0-92.0)$ \\
\hline $2 \mathrm{yr}$ & $82.8(78.2-87.7)$ & $89.7(86.4-93.1)$ & $85.8(81.2-90.7)$ & $82.5(78.7-86.5)$ \\
\hline $3 \mathrm{yr}$ & $80.4(74.9-86.3)$ & $86.1(81.7-90.7)$ & $76.9(69.2-85 \cdot 3)$ & $73.6(68.0-79.6)$ \\
\hline $4 \mathrm{yr}$ & $78.7(72.5-85.4)$ & $82.4(77.0-88.1)$ & $74.8(66.6-84.1)$ & $68.1(61.4-75.6)$ \\
\hline $5 \mathrm{yr}$ & $74.4(66.5-83.3)$ & $74.9(67.6-82.9)$ & $74.8(66.6-84.1)$ & $65.4(58.1-73.6)$ \\
\hline
\end{tabular}

CI, confidence interval; CTO, chronic total occlusion; POBA, plain old balloon angioplasty. 
Supplementary Table 2. Patients' baseline characteristics in the whole population

\begin{tabular}{|c|c|c|c|}
\hline Characteristic & $\operatorname{POBA}(\mathrm{n}=528)$ & Stenting $(\mathrm{n}=8 \mathrm{O} 1)$ & $p$ value \\
\hline Age & $68.68 \pm 8.68$ & $69.73 \pm 9.73$ & 0.043 \\
\hline Male sex & $392(74.2)$ & $656(81.9)$ & 0.001 \\
\hline BMI & $23.80 \pm 3.80$ & $23.28 \pm 3.28$ & 0.017 \\
\hline Hypertension & $414(78.4)$ & $606(75.7)$ & 0.273 \\
\hline $\mathrm{DM}$ & $364(68.9)$ & $464(57.9)$ & $<0.001$ \\
\hline Hypercholesterolemia & $209(39.6)$ & $330(41.2)$ & 0.596 \\
\hline Current smoker & $121(22.9)$ & $268(33 \cdot 5)$ & $<0.001$ \\
\hline $\mathrm{CHF}$ & $40(7.6)$ & $57(7.1)$ & 0.836 \\
\hline CAD & $313(59 \cdot 3)$ & $454(56.7)$ & 0.377 \\
\hline CKD & $157(29.7)$ & $150(18.7)$ & $<0.001$ \\
\hline Previous stroke & $94(17.8)$ & $117(14 \cdot 6)$ & 0.138 \\
\hline Previous bypass surgery & $56(10.6)$ & $61(7 \cdot 6)$ & 0.074 \\
\hline Previous amputation & $60(11.4)$ & $32(4 \cdot 0)$ & $<0.001$ \\
\hline Previous PTA & $83(15 \cdot 7)$ & $97(12.1)$ & 0.072 \\
\hline CLI & $233(44.1)$ & $238(29.7)$ & $<0.001$ \\
\hline Aspirin & $449(87.2)$ & $687(87 \cdot 3)$ & $>0.999$ \\
\hline Clopidogrel & $413(80.2)$ & $689(87.5)$ & $<0.001$ \\
\hline Cilostazol & $212(41.2)$ & $335(42.6)$ & 0.657 \\
\hline Statin & $361(70.1)$ & $574(72.9)$ & 0.294 \\
\hline
\end{tabular}

Values are presented as mean \pm SD or number (\%).

POBA, plain old balloon angioplasty; BMI, body mass index; DM, diabetes mellitus; CHF, congestive heart failure; CAD, coronary artery disease; CKD, chronic kidney disease; PTA, percutaneous transluminal angioplasty; CLI, critical limb ischemia. 
Supplementary Table 3. Lesion and procedural characteristics in the whole population

\begin{tabular}{|c|c|c|c|}
\hline Characteristic & $\operatorname{POBA}(\mathrm{n}=620)$ & Stenting $(\mathrm{n}=938)$ & $p$ value \\
\hline ABI target & $0.64 \pm 0.64$ & $0.57 \pm 0.57$ & $<0.001$ \\
\hline ABI nontarget & $0.81 \pm 0.26$ & $0.76 \pm 0.23$ & 0.008 \\
\hline Aortoiliac lesion & $78(12.6)$ & $272(29.0)$ & $<0.001$ \\
\hline Below-the-knee lesion & $126(45 \cdot 8)$ & $173(34.2)$ & 0.002 \\
\hline Diameter stenosis, \% & $89.40 \pm 12.62$ & $92.44 \pm 11.28$ & $<0.001$ \\
\hline Lesion length, mm & $141.80 \pm 105.84$ & $169.79 \pm 112.08$ & $<0.001$ \\
\hline Maximal balloon diameter, $\mathrm{mm}$ & $5.24 \pm 1.12$ & $5.60 \pm 2.49$ & 0.003 \\
\hline Balloon length, mm & $162.48 \pm 140.62$ & $130.11 \pm 109.66$ & $<0.001$ \\
\hline Max stent diameter, mm & & $6.85 \pm 2.94$ & \\
\hline Stent length sum, mm & & $129.86 \pm 78.34$ & \\
\hline Technical success & & & $<0.001$ \\
\hline No & $29(4.8)$ & $24(2.6)$ & \\
\hline Sub-optimal & $62(10.2)$ & $27(2.9)$ & \\
\hline Yes & $518(85.1)$ & $875(94 \cdot 5)$ & \\
\hline Good antegrade flow & $573(94.1)$ & $890(96.1)$ & 0.087 \\
\hline Residual stenosis, $<30 \%$ & $521(85.6)$ & $887(95.8)$ & $<0.001$ \\
\hline Contrast volume, $\mathrm{mL}$ & $168.19 \pm 84.42$ & $172.78 \pm 96.36$ & 0.396 \\
\hline Clinical success & $336(54.2)$ & $663(70.7)$ & $<0.001$ \\
\hline Post-ABI & $0.83 \pm 0.23$ & $0.85 \pm 0.18$ & 0.133 \\
\hline
\end{tabular}

Values are presented as mean \pm SD or number $(\%)$.

POBA, plain old balloon angioplasty; ABI, ankle-brachial index. 


\section{KJIM}

Supplementary Table 4. Patients baseline characteristics according to TASC II AB vs. CD

\begin{tabular}{|c|c|c|c|c|c|c|}
\hline \multirow[b]{2}{*}{ Characteristic } & \multicolumn{3}{|c|}{$\operatorname{TASC}(\mathrm{A}, \mathrm{B})(\mathrm{n}=78 \mathrm{l})$} & \multicolumn{3}{|c|}{$\operatorname{TASC}(\mathrm{C}, \mathrm{D})(\mathrm{n}=827)$} \\
\hline & $\begin{array}{c}\text { POBA } \\
(n=255)\end{array}$ & $\begin{array}{l}\text { Stenting } \\
(\mathrm{n}=288)\end{array}$ & $p$ value & $\begin{array}{c}\text { POBA } \\
(n=306)\end{array}$ & $\begin{array}{l}\text { Stenting } \\
(\mathrm{n}=521)\end{array}$ & $p$ value \\
\hline Age & $67.72 \pm 7.72$ & $69.53 \pm 9.53$ & 0.027 & $69.39 \pm 9.39$ & $69.85 \pm 9.85$ & 0.499 \\
\hline Male sex & $173(76.9)$ & $239(83.0)$ & 0.107 & $219(72.3)$ & $417(81.3)$ & 0.004 \\
\hline BMI & $23.93 \pm 3.93$ & $23.46 \pm 3.46$ & 0.165 & $23.70 \pm 3.70$ & $23.18 \pm 3.18$ & 0.067 \\
\hline Hypertension & $188(83.6)$ & $228(79.2)$ & 0.252 & $226(74.6)$ & $378(73.7)$ & 0.840 \\
\hline $\mathrm{DM}$ & $175(77.8)$ & $176(61.1)$ & $<0.001$ & $189(62.4)$ & $288(56.1)$ & 0.094 \\
\hline Hypercholesterolemia & $102(45 \cdot 3)$ & $148(51.4)$ & 0.203 & $107(35 \cdot 3)$ & $182(35 \cdot 5)$ & $>0.999$ \\
\hline Current smoker & $49(21.8)$ & $79(27 \cdot 4)$ & 0.172 & $72(23.8)$ & $189(36.8)$ & $<0.001$ \\
\hline $\mathrm{CHF}$ & $13(5.8)$ & $24(8.3)$ & 0.348 & $27(8.9)$ & $33(6.4)$ & 0.241 \\
\hline CAD & $145(64.4)$ & $179(62.2)$ & 0.659 & $168(55 \cdot 4)$ & $275(53.6)$ & 0.662 \\
\hline CKD & $77(34.2)$ & $67(23 \cdot 3)$ & 0.008 & $80(26.4)$ & $83(16.2)$ & 0.001 \\
\hline Previous stroke & $43(19.1)$ & $51(17 \cdot 7)$ & 0.770 & $51(16.8)$ & $66(12.9)$ & 0.145 \\
\hline Previous bypass surgery & $33(14.7)$ & $26(9.0)$ & 0.065 & $23(7 \cdot 6)$ & $35(6.8)$ & 0.786 \\
\hline Previous amputation & $23(10.2)$ & $9(3.1)$ & 0.002 & $37(12.2)$ & $23(4 \cdot 5)$ & $<0.001$ \\
\hline Previous PTA & $27(12.0)$ & $31(10.8)$ & 0.766 & $56(18.5)$ & $66(12.9)$ & 0.038 \\
\hline CLI & $97(43.1)$ & $66(22.9)$ & $<0.001$ & $136(44 \cdot 9)$ & $172(33.5)$ & 0.002 \\
\hline Aspirin & $196(90.7)$ & $258(92.5)$ & 0.597 & $253(84.6)$ & $429(84.4)$ & $>0.999$ \\
\hline Clopidogrel & $184(85.2)$ & $249(89.2)$ & 0.224 & $229(76.6)$ & $440(86.6)$ & $<0.001$ \\
\hline Cilostazol & $80(37.0)$ & $107(38.4)$ & 0.837 & $132(44.1)$ & $228(44.9)$ & 0.897 \\
\hline Statin & $168(77.8)$ & $211(75.6)$ & 0.650 & $193(64.5)$ & $363(71.5)$ & 0.049 \\
\hline
\end{tabular}

Values are presented as mean $\pm \mathrm{SD}$ or number (\%).

TASC, TransAtlantic Inter-Society Consensus classification; POBA, plain old balloon angioplasty; BMI, body mass index; DM, diabetes mellitus; CHF, congestive heart failure; CAD, coronary artery disease; CKD, chronic kidney disease; PTA, percutaneous transluminal angioplasty; CLI, critical limb ischemia. 
Supplementary Table 5. Lesion and procedural characteristics according to the presence of chronic total occlusion

\begin{tabular}{|c|c|c|c|c|c|c|}
\hline \multirow{2}{*}{ Characteristic } & \multicolumn{3}{|c|}{$\operatorname{TASC}(\mathrm{A}, \mathrm{B})(\mathrm{n}=78 \mathrm{1})$} & \multicolumn{3}{|c|}{$\operatorname{TASC}(\mathrm{C}, \mathrm{D})$} \\
\hline & $\operatorname{POBA}(n=262)$ & Stenting $(\mathrm{n}=333)$ & $p$ value & $\operatorname{POBA}\left(\mathrm{n}=35^{8}\right)$ & Stenting $(n=605)$ & $p$ value \\
\hline ABI target & $0.72 \pm 0.26$ & $0.64 \pm 0.20$ & 0.001 & $0.58 \pm 0.22$ & $0.52 \pm 0.20$ & 0.001 \\
\hline ABI nontarget & $0.85 \pm 0.25$ & $0.81 \pm 0.21$ & 0.126 & $0.78 \pm 0.26$ & $0.73 \pm 0.24$ & 0.035 \\
\hline Aortoiliac lesion & $36(13.7)$ & $83(24 \cdot 9)$ & 0.001 & $42(11.7)$ & $189(31.2)$ & $<0.001$ \\
\hline Below-the-knee lesion & $142(54.2)$ & $87(26.1)$ & $<0.001$ & $186(52.0)$ & $216(35 \cdot 7)$ & $<0.001$ \\
\hline TASC & & & 0.166 & & & 0.916 \\
\hline A & $100(38.2)$ & $147(44.1)$ & & & & \\
\hline $\mathrm{B}$ & $162(61.8)$ & $186(55 \cdot 9)$ & & & & \\
\hline $\mathrm{C}$ & & & & $163(45 \cdot 5)$ & $272(45.0)$ & \\
\hline $\mathrm{D}$ & & & & $195(54 \cdot 5)$ & $333(55 \cdot 0)$ & \\
\hline Diameter stenosis, \% & $83.49 \pm 13.28$ & $86.43 \pm 13.22$ & 0.007 & $93.73 \pm 10.14$ & $95.75 \pm 8.40$ & 0.002 \\
\hline Lesion length, cm & $81.05 \pm 65.05$ & $84.96 \pm 57.43$ & 0.470 & $186.35 \pm 107.87$ & $216.02 \pm 107.49$ & $<0.001$ \\
\hline Maximal balloon diameter, $\mathrm{mm}$ & $5.18 \pm 1.35$ & $5 \cdot 51 \pm 2.27$ & 0.035 & $5.29 \pm 0.90$ & $5.65 \pm 2.61$ & 0.003 \\
\hline Balloon length, mm & $147 \cdot 30 \pm 36.51$ & $109.81 \pm 107.24$ & 0.001 & $174.12 \pm 142.82$ & $141.44 \pm 109.45$ & 0.001 \\
\hline Max stent diameter, mm & & $6.79 \pm 3.23$ & & & $6.89 \pm 2.78$ & \\
\hline Stent length sum, mm & & $100.65 \pm 56.57$ & & & $145.17 \pm 83.67$ & \\
\hline Technical success & & & 0.001 & & & $<0.001$ \\
\hline No & $4(1.6)$ & $5(1.5)$ & & $25(7.1)$ & $19(3.2)$ & \\
\hline Sub-optimal & $29(11.3)$ & $11(3 \cdot 3)$ & & $33(9 \cdot 4)$ & $16(2.7)$ & \\
\hline Yes & $224(87.2)$ & $315(95 \cdot 2)$ & & $294(83 \cdot 5)$ & $560(94.1)$ & \\
\hline Good antegrade flow & $248(96.5)$ & $321(97 \cdot 0)$ & 0.927 & $325(92.3)$ & $569(95 \cdot 6)$ & 0.047 \\
\hline Residual stenosis, $<30 \%$ & $228(88.7)$ & $322(97 \cdot 3)$ & $<0.001$ & $293(83.2)$ & $565(95.0)$ & $<0.001$ \\
\hline Contrast volume, $\mathrm{mL}$ & $162.85 \pm 8.32$ & $156.81 \pm 102.70$ & 0.497 & $172.26 \pm 81.26$ & $182.91 \pm 90.78$ & 0.125 \\
\hline Clinical success & $145(55 \cdot 3)$ & $241(72.4)$ & $<0.001$ & $191(53.4)$ & $422(69.8)$ & $<0.001$ \\
\hline Post-ABI & $0.88 \pm 0.21$ & $0.89 \pm 0.17$ & 0.554 & $0.79 \pm 0.24$ & $0.83 \pm 0.19$ & 0.082 \\
\hline
\end{tabular}

Values are presented as mean \pm SD or number (\%).

TASC, TransAtlantic Inter-Society Consensus classification; POBA, plain old balloon angioplasty; ABI, ankle-brachial index. 

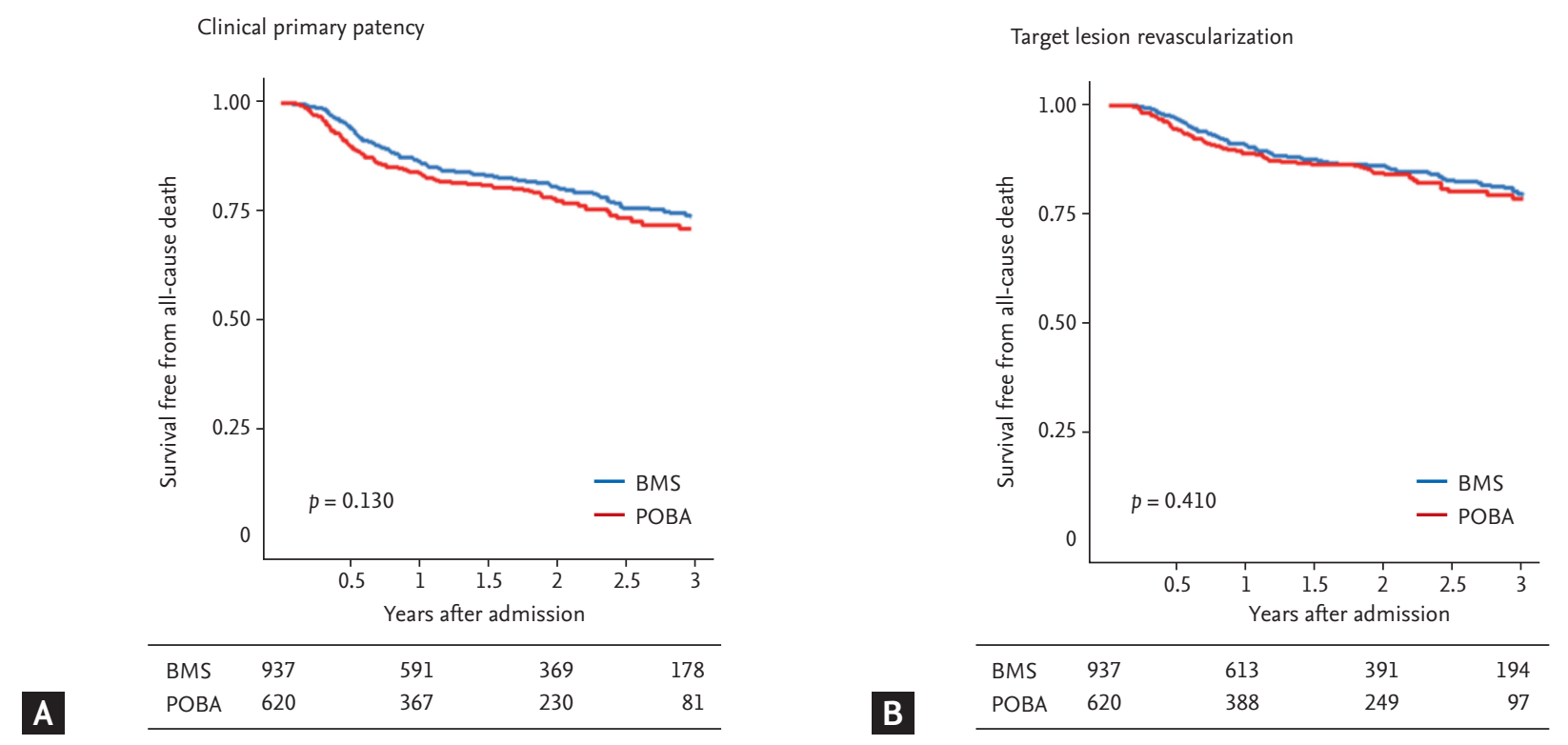

Supplementary Figure 1. (A) Clinical primary patency. (B) Target lesion revascularization. BMS, bare metal stent; POBA, plain old balloon angioplasty. 
TASC AB

Clinical primary patency

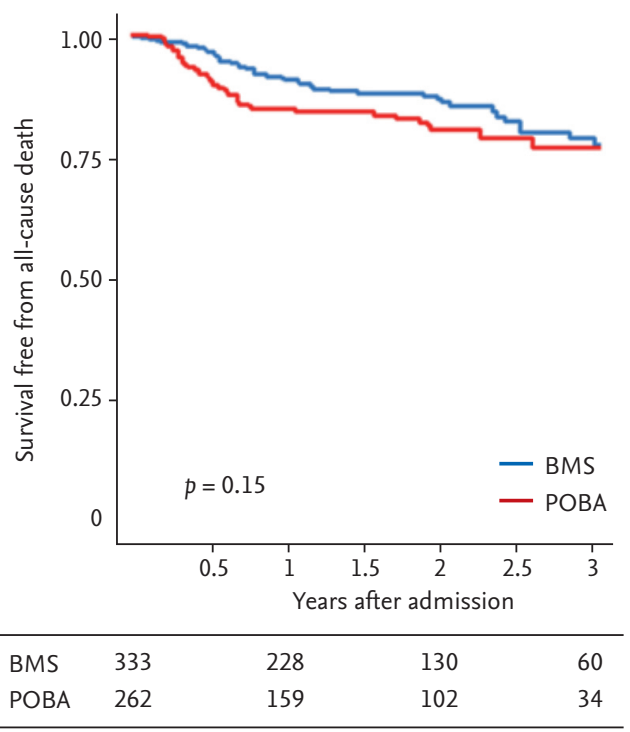

Freedom from TLR

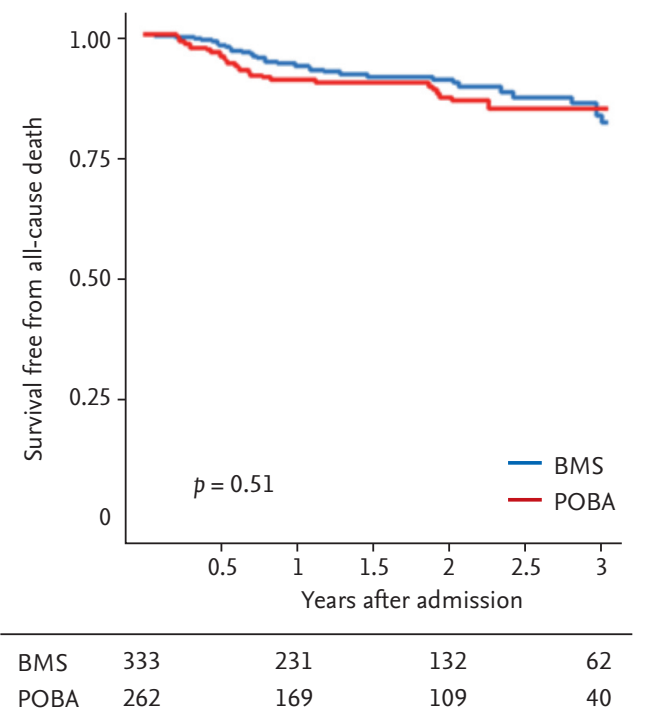

TASC CD
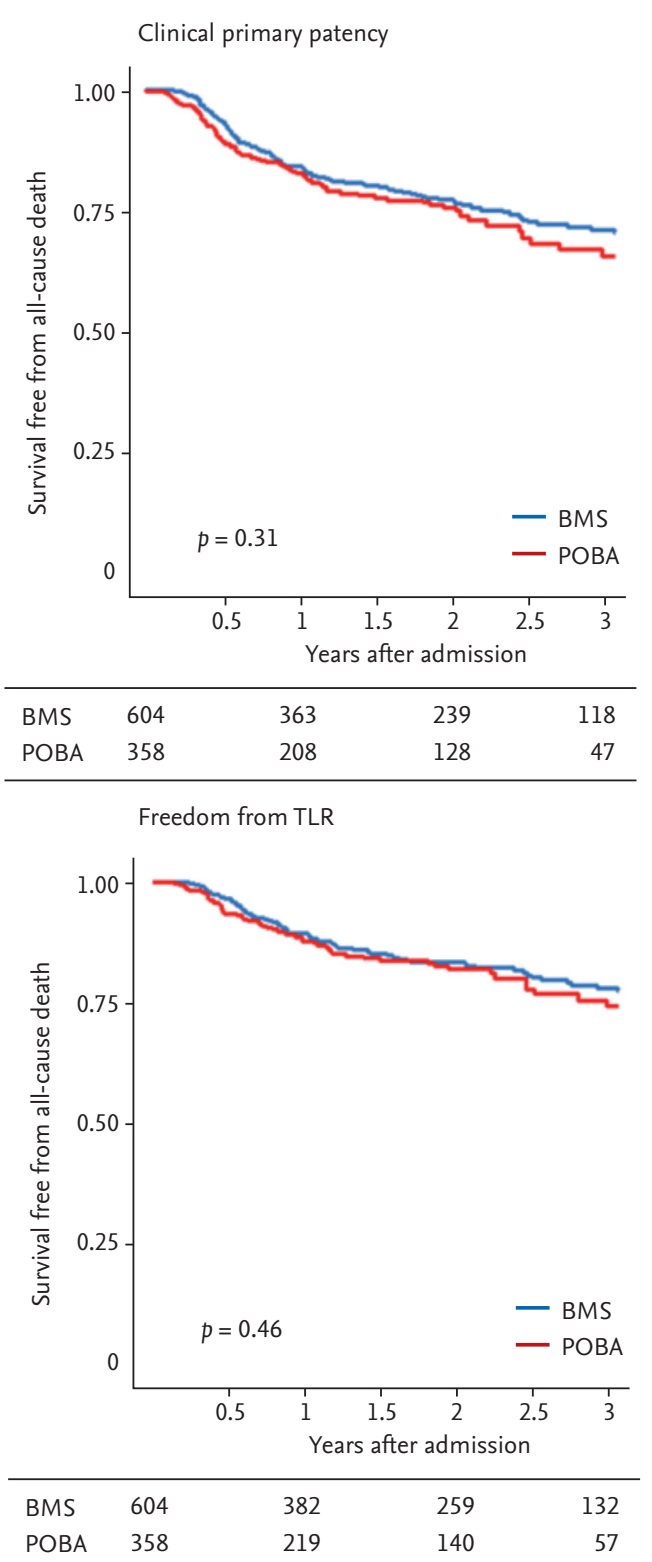

Supplementary Figure 2. Kaplan-Meier curves for outcomes according to TransAtlantic Inter-Society Consensus classification (TASC) AB vs. CD. (A) Clinical primary patency. (B) Clinical primary patency. (C) Freedom from target lesion revascularization (TLR). (D) Freedom from TLR. BMS, bare metal stent; POBA, plain old balloon angioplasty. 

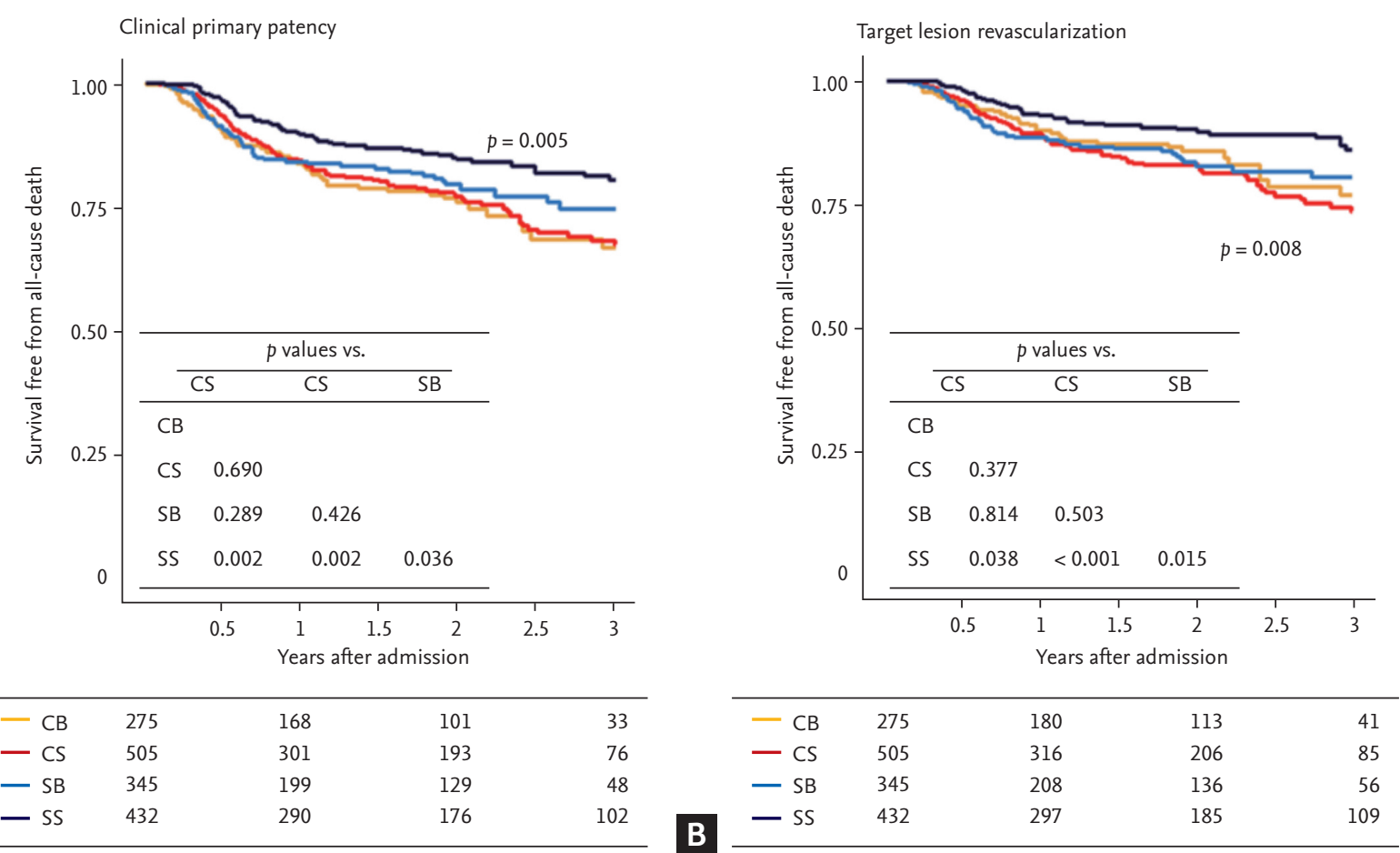

Supplementary Figure 3. Kaplan-Meier curves for outcomes in various subgroups: de novo lesion only. (A) Clinical primary patency, (B) target lesion revascularization. CB, patient group with chronic total occlusion treated by plain balloon angioplasty; CS, patient group with chronic total occlusion treated by stenting; SB, patient group without chronic total occlusion treated by plain balloon angioplasty; SS, patient group without chronic total occlusion treated by stenting. 
Clinical primary patency

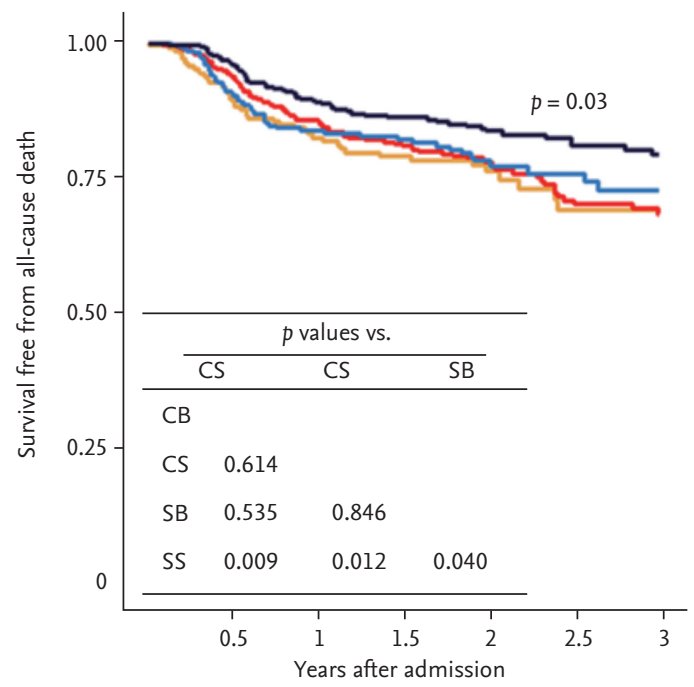

\begin{tabular}{rrrrr}
\hline - CB & 214 & 130 & 78 & 28 \\
- CS & 465 & 279 & 180 & 72 \\
- SB & 304 & 169 & 104 & 40 \\
- SS & 406 & 271 & 165 & 98 \\
\hline
\end{tabular}

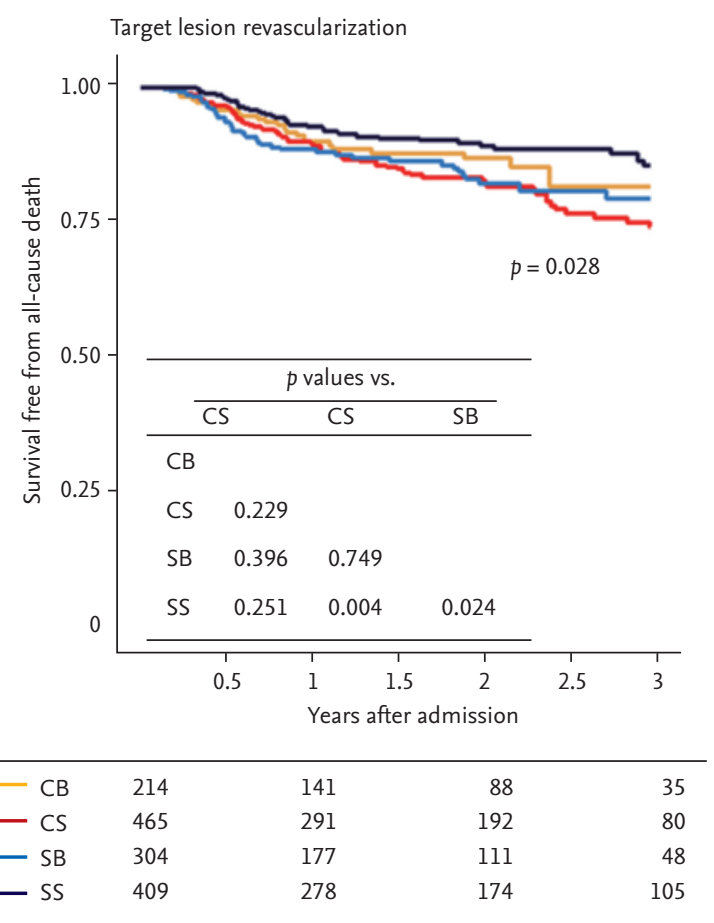

Supplementary Figure 4. Kaplan-Meier curves for outcomes in various subgroups: technical success only. (A) Clinical primary patency, (B) target lesion revascularization. CB, patient group with chronic total occlusion treated by plain balloon angioplasty; CS, patient group with chronic total occlusion treated by stenting; SB, patient group without chronic total occlusion treated by plain balloon angioplasty; SS, patient group without chronic total occlusion treated by stenting. 

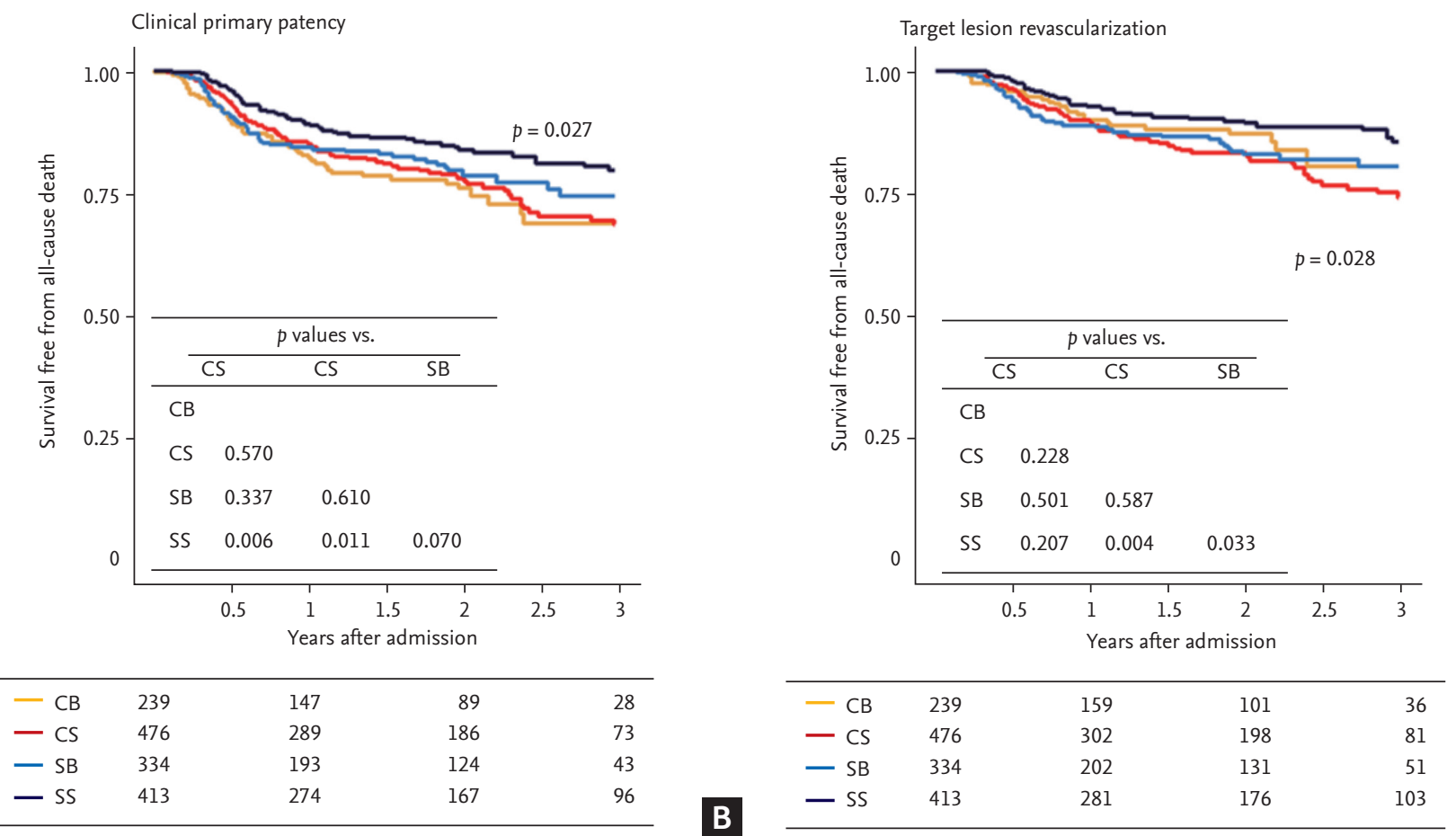

Supplementary Figure 5. Kaplan-Meier curves for outcomes in various subgroups: good antegrade flow only. (A) Clinical primary patency, (B) target lesion revascularization. CB, patient group with chronic total occlusion treated by plain balloon angioplasty; CS, patient group with chronic total occlusion treated by stenting; SB, patient group without chronic total occlusion treated by plain balloon angioplasty; SS, patient group without chronic total occlusion treated by stenting. 